\title{
Gap-Dependent Microrheometry of Complex Liquids
}

\author{
Dr. Christian Clasen \& Prof. Gareth H. McKinley \\ Hatsopoulos Microfluids Laboratory \\ Dept. Of Mechanical Engineering \\ Massachusetts Institute of Technology \\ Cambridge,MA 02139, USA
}

June 6, 2003

Plenary paper presented at UWINNFM Workshop Rheometry II

Miskin Manor, April 2003

\begin{abstract}
We review recent developments in the field of microrheometry and describe a new flexurebased microrheometer (FMR) that can make measurements of the steady shear viscosity of transparent or optically-opaque complex fluids using 1-10 $\mu 1$ samples. We document the complex microrheology that may arise in a typical consumer product such as a skin crème, when the microstructure of the multiphase material (in this case a oil-water emulsion containing microcrystalline wax particles) and the characteristic length scale of the flow device become comparable. The material is a highly filled emulsion and conventional cone-and-plate rheometry shows that the material exhibits an apparent yield stress. Analogous tests in the microrheometer show that a sequence of flow transitions occur which lead to an apparent viscosity that is both gapand shear-stress- (or shear-rate)-dependent. The microrheological measurements are combined with a slip analysis and optical microscopy to formulate a 'phase diagram' identifying the critical stress and corresponding slip velocity for each flow transition as a function of the gap. In the case of the skin crème investigated in the present study, the flow behavior in small gaps below $100 \mu \mathrm{m}$ is dominated by the presence of micro-crystalline wax particles with mean diameters in the range of $50 \mu \mathrm{m}$. The sudden decrease in the apparent viscosity, or equivalently the sudden increase in the slip velocity between two surfaces with decreasing gap and increasing applied stress is of importance in end-user perceptions of textural quantities such as 'slipperiness' or 'lubricity' which are typically used to characterize creams and other complex consumer products. The ability to progressively vary the gap between the two shearing surfaces means that the flexure-based microrheometer developed in the present work may find future applications in helping to bridge the gap between the traditionally-distinct fields of bulk rheological characterization and tribological testing.
\end{abstract}




\section{Introduction}

The term microrheometry has been employed by numerous authors to refer to a large number of different experimental configurations. In general, the term may be best defined as the science of measuring, quantitatively, the rheological properties of a fluid sample when one characteristic dimension is on the scale of order microns. At such scales, the geometric dimension, the microstructural features of the fluid and possibly the characteristic roughness of the test fixtures can all begin to interact. An overview of different instrumental approaches to microrheometry is provided in Figure 1 and discussed below.

At the coarsest level we may differentiate between bulk microrheometry and interfacial rheometry, which by definition involves deformations in domains with a characteristic lateral thickness of a few molecular dimensions. Static and dynamic properties of fluid interfaces - such as the disjoining pressure $\Pi$, interfacial tension, spreading coefficient $S$, and contact angle $\theta$ - can be measured using traditional Langmuir troughs and ring or plate interfacial tensiometers. In addition the viscous and elastic properties of interfaces undergoing deformation at controlled rates can now be quantified using interfacial rheometers $[1,2]$. The topic of interfacial rheometry and analysis of common instrumental configurations are covered in detail in the treatise by Edwards et al. [3].

As indicated in the figure, the field of tribology may be considered to sit at the junction of bulk and interfacial rheology, since measurements of the 'lubricity' or coefficient of sliding friction $(\mu)$ contain information about both the rheological properties of the fluid and the physicochemical properties of the bounding surfaces [4]. In recent years a number of new quantitative techniques, based on the Atomic Force Microscope (AFM) and Sliding Force Apparatus (SFA) have been developed to quantify microscopic features of fluid lubrication (see for example [4]). For rough surfaces, the theory of elastohydrodynamic lubrication incorporates both asperity contact and the viscous or viscoelastic properties of the lubricant [5]. When the test surfaces are atomically smooth, (such as the opposed cylindrical sheets of cleaved mica utilized in the SFA) and the gaps are reduced to the nanometer scale, esoteric effects such as local crystalline ordering in the fluid may arise. These phenomena are beyond the scope of this discussion; however they have been referred to collectively as "nanorheology" and have been studied extensively by Granick and coworkers $[6,7]$. For standard machine-finished surfaces such extreme confinement effects 
are not expected to be present due to onset of asperity contacts when the gap separation becomes on the order of the mean roughness.

Bulk microrheology has evolved rapidly over the past decade as the ability to fabricate devices, observe submicron motion and quantify deformations on the microscale have developed. The instrumentation may be conveniently subdivided into 'active' devices that impose a controlled deformation and 'passive' techniques that rely on random forcing from Brownian motion of colloidal tracers dispersed in the sample. These latter particulate probe techniques have been pioneered by researchers in the physics community including D. Weitz, D. Pine and coworkers, and have recently been reviewed by [8] and [9]. The methods of analysis depend on the number density of particulate probes present in the sample. In the multiple scattering limit, Diffusing Wave Spectroscopy (DWS) can be used to follow the decay in the autocorrelation of a coherent laser beam passed through a sample [10]. Originally developed for forward scattering, recently a backscattering technique has been developed which enables the use of a fiber optic probe and which can analyze very small sample volumes of as little as 0.1 picoliter [11].

If only a single particle is introduced, then laser-induced fluorescence and image processing techniques can be used to track, with subpixel accuracy, the random walk path followed by the centroid of the colloidal probe as it diffuses through the fluid matrix under the action of thermal fluctuations. It is important to note that the submicron probes introduced in these techniques may perturb the local equilibrium conformation and resulting rheological properties of the medium to be measured, so that one is in effect measuring the properties of a new effective microenvironment. This is indicated graphically in Figure 1 by the two images taken from [12]. The left-hand panel shows an electron microscope image of a $1 \mu \mathrm{m}$ polystyrene (PS) bead surround by the local mesh of an F-Actin biopolymer gel. Laser Tracking Microrheology measurements (image B) of the beads showed that the r.m.s. displacement observed over a period of 30 seconds varied by more than an order of magnitude depending on whether the PS bead was coated with a protein (Bovine Serum Albumen, BSA-PS) or a carboxylated surface (COO-PS). The effective viscoelastic properties of the gel computed from these two trajectories show corresponding differences. Most recently a two-fluid cross-correlative technique has been proposed by [13, 14] to help overcome unwanted local changes in the microenvironment near the particle surface. 
Regardless of the particular particle tracking methodology chosen, these passive microrheometry techniques require the use of a generalized Stokes-Einstein relationship to convert the observed measurements of particle motion into the conventional frequency-dependent viscoelastic functions, $G^{\prime}(\omega)$ and $G^{\prime \prime}(\omega)$ [9, 15]. These techniques are thus limited to measurements of the linear viscoelastic properties of the complex fluid; nevertheless they use very small sample volumes and can span a much wider range of frequencies than conventional macrorheological oscillatory devices.

Externally-driven or 'active' microrheological techniques use displacements imposed at the boundary to control the deformation of the complex fluid being probed and are thus more closely related to macroscopic rheometers. They may be subdivided loosely into shear and extensional devices.

Atomic force microscopes form a useful platform for developing microrheological techniques. The tip may be brought into contact with a surface and then pulled away in order to measure contact forces and adhesion ([16]). Alternatively in simple liquids microscale cavitation effects can be measured [17]. By chemically binding macromolecules such as DNA or synthetic polymers to the AFM tip and also to the opposing surface, the equilibrium force-extension curves for individual chains can even be measured; a general area known as molecular force microscopy (see for example [18]). Finally, we note that the effective extensional viscosity of micron-scale threads of complex fluids such as saliva, silk threads and other biopolymers can also be measured using a microscale version of the 'capillary break-up rheometer' [19, 20]. We have recently used this device in conjunction with the flexure-based microrheometer in order to make ex-vivo measurements of microliter samples of viscoelastic spider silk solutions which contain ca. $35 \%$ protein [21].

In the oscillating AFM probe device, shifts in the resonant frequency and damping of a tipless AFM cantilever are measured. These result from the viscous or linear viscoelastic properties of the surrounding medium and used to accurately evaluate the shear viscosity of the fluid in the local environment of the cantilever [22]. Although the flow is, in some sense, boundary-driven, this technique shares a number of logical connections with the particulate probes discussed above, as it is also a 'passive' rather than a 'driven' technique and the damped harmonic motion of the cantilever must be interpreted in terms of either a Newtonian or linear viscoelastic fluid model. 
In the present paper we utilize a shearing microrheometer based on a precision-machined compound flexure to impose large amplitude shear displacements on a sample confined between two optical flats [23]. The instrument is a strain-controlled device that generates a plane Couette flow in the sample. If the spring constant of the upper flexure is known through calibration, then the measured deflections of the upper surface can be converted to yield the shear stress acting on the sample. The device is thus essentially a miniaturized sliding plate rheometer of the same type as those developed by Dealy and coworkers for the study of polymer melts [24] but which utilizes less than $5 \mu 1$ of fluid. A similar instrument has been developed by Dhinojwala \& Granick [25]. Further details of our instrument design are documented in the Experimental section below.

In addition to the ability of microrheometry to probe the properties of very small samples of complex fluids, it can be used to explore systematic changes in the effective rheological properties of a multiphase material as the degree of geometric confinement is systematically increased. In many systems, the rheological properties that are measured utilizing a bulk sample in a conventional rheometer do not adequately represent the flow behavior of a complex fluid in the more extreme conditions that can occur during processing or the final application. This is especially true for the rheological properties of healthcare and consumer products such as liquid or gelled foods or lotions and skin crèmes when the microscopic structure of the filled emulsion reaches the dimensions of the flow geometries during the spreading and application processes of the final product $[26,27]$.

In our previous paper, we have documented gap-dependent stress levels for different yielding and slipping processes in mayonnaise. By contrast, control experiments with rheologically-simple polymeric fluids such as a low molecular weight unentangled polybutadiene melt showed no gap-dependence as expected. The trends documented in the gap-dependent microrheological observations could be understood in terms of a "phase diagram" for the flow behavior in small gaps that directly correlates the stress levels required for certain deformation mechanisms with the size of the gap and the microstructural components of the fluid [23]. Similar evidence of a gap-dependent yield stress for suspensions and emulsions in small gaps has been reported by Meeten in squeeze flow experiments [28-30]. However the microrheological behavior of many other complex fluids has not yet been investigated. The aim of this paper is therefore to report on a detailed investigation of the 'lubricity' - i.e. the flow behavior in small gaps - of 
another complex fluid, a commercially-available skin crème product, using the flexure based microrheometer (FMR).

\section{Experimental}

The Flexure-based Microgap Rheometer (FMR) is shown in Figure 2. It consists of a miniaturized sliding plate rheometer that generates a plane Couette shearing flow between two optical flats (polished flat to within $\lambda / 20$, or $30 \mathrm{~nm}$ and coated with a semi-reflective layer of $100 \mathrm{~nm}$ $\left.\mathrm{TiO}_{2}\right)$.

One key element of the FMR is the capability of delivering large deformations, whilst also maintaining a constant gap separation with submicron resolution. This is achieved using a compound flexure system [31]. This compound flexure is used to hold the test fixtures and the fluid sample between a drive spring and an independent sensor spring. The flexure system compensates for orthogonal as well as for rotational displacements and enables purely linear relative translation of the surfaces over distances of several millimeters (controlled by an "inchworm" nanomotor) with an orthogonal displacement of less than $1 \mathrm{~nm}$. Alignment fidelity, device orthogonality and total error stack-up are all optimized by machining the entire instrument frame from a single monolithic aluminum block using CAD design plus water-jet and EDM technology. Displacements in the sensing flexure are detected using an inductive proximity sensor with a resolution of $\pm 3 \mathrm{~nm}$. The spring constant of the design utilized in the present work is determined by calibration to be $8.2 \times 10^{4} \mathrm{~N} / \mathrm{m}$.

The parallelism of the shearing surfaces with respect to the shearing direction, as well as the absolute gap separation, are both measured and controlled using white light interferometry [25, 32]. A semi-reflective coating of $\mathrm{TiO}_{2}$ is applied to the optical flats which are then brought into close proximity in order to form the bounding surfaces of the fixture. Only integer wavelengths of light are passed through the resulting optical cavity that is formed between the bounding plates. Orthogonal 1D optical crosscuts of the shearing surfaces are dispersed by a spectrometer. Image processing of the fringe pattern allows the determination of the tilt angle of the surfaces and simultaneous feedback adjustment to the parallelism is performed using software-controlled nano- 
positioning stages. The absolute gap $h$ can be calculated in terms of the known refractive index of the fluid $\left(n_{f}\right)$ and from two fringe wavelengths $\lambda_{m}$ and $\lambda_{m+k}$ according to the following equation:

$$
h=\frac{k}{2 \cdot n_{f}} \cdot \frac{\lambda_{m} \cdot \lambda_{m+k}}{\lambda_{m}-\lambda_{m+k}}, \quad k=1,2 \ldots
$$

The gap can be adjusted with a single nano-positioning stage with a step resolution of $\Delta h<$ $30 \mathrm{~nm}$. In addition to direct optical control of the sample gap, the determination of the relative position of the upper surface can also be measured independently using a second inductive proximity sensor. This allows accurate determination of the gap for complex fluid systems that are not optically transparent (such as the skin crème investigated in this paper) with a resolution of $\pm 10 \mathrm{~nm}$.

In an ambient laboratory environment that is not dust free, our preliminary calibration experiments have determined that the minimum practical gap for experiments is limited to $h_{\min } \sim$ $5 \mu \mathrm{m}$. Below this minimum gap setting trapped dust particles dominate the stress response below a (fluid-dependent) critical shear rate and cause a nearly-constant sliding frictional stress that is characteristic of tribological measurements of the coefficient of sliding friction. A similar flexurebased device has also been designed for systematically performing such measurements [33]. More detailed description of the instrumental setup, its range and limitations are given in [23]. The viscometric properties of bulk samples were also characterized using a controlled stress rheometer (AR1000, TA Instruments, New Castle, DE 19720, USA) with a conventional cone-and-plate system $\left(\varnothing=40 \mathrm{~mm}, 1^{\circ} 59^{\prime}, 59 \mu \mathrm{m}\right.$ cone truncation). The specific skin crème investigated is a commercially available product (further details of which are available upon request from the authors). Rheologically similar materials are discussed by Brummer and Godersky [26].

\section{Results and discussion}

\section{Gap-Independent Microrheometry}

In order to be able to unambiguously interpret the gap-dependence of microrheometric observations in complex fluids, it is first necessary to ensure that 'simple' polymer solution and 
melt systems show the same viscometric properties on a microscopic scale as the corresponding bulk sample. Figure 3 shows micro-rheological data for a dilute polystyrene solution with gaps ranging from $59.9 \mu \mathrm{m}$ to $3.4 \mu \mathrm{m}$ together with the comparable bulk observations. The shear stresses measured using the microrheometer superpose for all gap settings that have been used. The systematic upturn of the shear stress for the lowest gap setting of $3.4 \mu \mathrm{m}$ is a result of the sliding friction of trapped dust particles. This regime is typically referred to in the tribological literature as 'boundary lubrication' between asperities or point contacts. At higher shear-rates, hydrodynamic lubrication forces dominate these contact forces and the microrheometry data for $3.4 \mu \mathrm{m}$ superposes with the measurements for other gap settings in which there are no frictional contacts between the two plates of the microrheometer. If the normal contact force is also known (which it is not in the present design) then such rheological data can also be represented in the form of a so-called 'Stribeck curve' [34].

A similar gap invariance is demonstrated in figure 4 for microrheological measurements of the viscosity of a standard unentangled PDMS melt. The measured viscosity is nearly at the lowest resolution limit of the present design of the microrheometer. Since the lowest sensitivity of the device depends on the stress acting on the sensor spring, the reproducibility of the viscosity of this standard system is only satisfying above a shear rate of $10 \mathrm{~s}^{-1}$, giving a lowest resolution limit for the shear stress of $10 \mathrm{~Pa}$ for the present design when the area of the shearing surface is $25 \mathrm{~mm}^{2}$. This minimum resolution of the shear stress can be adjusted by choosing a different spring constant of the sensor spring or by using a geometry with a different area of the shearing surface.

\section{Gap-Dependent Microrheology of Skin Crème}

Skin crèmes are typically highly filled emulsions containing complex mixtures of surfactants and fatty amphiphiles for long shelf-live and controlled rheological properties of the product [35]. Although there are many attempts to correlate the macroscopic rheological properties with the complex composition of the emulsion [36,37], there are only a few examples of attempts to correlate microscopic phenomena such as "skin feeling" during the application process of a thin film of skin creme with conventional "bulk" rheological parameters [26]. 
Conventional cone-and-plate measurements of the macroscopic or bulk rheology of the skin crème sample are shown in Figure 5 by the solid line. Note that we represent the viscosity as a function of the shear stress in order to better highlight the dramatic change in the rheology at a critical stress or 'yield stress' [38]. The bulk viscosity shows a large and essentially constant value of $\eta_{0} \approx 5 \times 10^{6} \mathrm{~Pa}$.s at low stresses, which then drops by approximately a factor of a thousand at a stress level of $\tau_{y} \approx 250 \pm 50 \mathrm{~Pa}$. We refer to this as a 'primary yield stress'. Standard creep measurements below this critical stress show that the material acts as a strongly viscoelastic liquid. Above the primary yield stress, the viscosity shows a progressively increasing shear thinning behavior that asymptotes beyond $725 \mathrm{~Pa}$ into a shear thinning region with a viscosity depending on the stress to a power of -5.6 (corresponding to a power law exponent for viscosity vs. shear rate of -0.85).

The microscopic flow behavior of the skin crème is also shown in Figure 5 and the data exhibits a pronounced gap-dependence. Note that the measurements for gaps below and above $55 \mu \mathrm{m}$ are offset onto two different stress axes as indicated by the arrows in order to clarify the systematic nature of the effects of the gap on the stress dependence of the viscosity. The bulk flow curve and the data for $55 \mu \mathrm{m}$ are shown on both the lower and upper abscissae for reference. It can be seen that for gaps above $55 \mu \mathrm{m}$, the samples still exhibit a primary yield stress at low deformation rates. However, the value of this primary yield stress increases with a decreasing gap. These measured values are transcribed below to a "phase diagram" of the same type as proposed by Clasen \& McKinley [23] for another highly filled consumer product: mayonnaise (cf. Figure 8 below). A comparison of the critical stress observed in microrheological measurements suggests that the critical stress level measured for the bulk sample using a conventional cone-and-plate rheometer could be an appropriate average of the values determined for the different micro gap settings.

However, for gaps below $55 \mu \mathrm{m}$, measurements in the FMR show that the primary yield stress can no longer clearly be detected. Instead, the viscosity shows a continuous power-law-like behavior at low values of the stress. This flow region at low stresses and small gaps also shows increased fluctuations in the data, as indicated by the upper and lower bounds on the plotted symbols. Despite these fluctuations, the average values for a certain gap show a good reproducibility from sample to sample. 
Independent of the specific gap setting, each of the flow curves shows a second, reproducible yielding region at a shear stress of $380 \mathrm{~Pa} \leq \tau \leq 400 \mathrm{~Pa}$ when the viscosity again decreases rapidly for small changes in the applied stress. For any constant value of the stress above this second yielding region the viscosity decreases monotonically with a decreasing gap, strongly suggesting that the nature of this second "pseudo" yielding region is in fact the onset of a slip flow [39]. We analyze this slip in detail below and the critical stress values for this transition at each different microgap setting are also transcribed to the "phase diagram" presented below in Figure 8.

Finally, at stresses of $\sim 500 \mathrm{~Pa}$ another change in the structure of the flow can be observed in the data at larger gaps. Below a gap of $44 \mu \mathrm{m}$, however, this third yielding region is smeared out and a simple inflexion point in each flow curve is observable.

\section{Analysis of Gap- and Stress-Dependent Slip}

The complex sequence of transitions shown in a curve of the apparent viscosity as a function of shear stress suggests that slip between the sample and the bounding plates may be important. This can be understood more clearly through a closer examination of the data.

A method for extracting the thickness of the true slip layer thickness $\delta$ for a certain stress was described by Yoshimura and Prud'homme [40, 41] following earlier work by Mooney. The analysis considers the actual deformation in the sample to be composed of a linear summation of a flow in the bulk with shear rate $\dot{\gamma}_{\mathrm{b}}$ and a second, higher shear rate in a slip layer $\dot{\gamma}_{\mathrm{w}}$, near each of the bounding walls. For slip layers with $\delta$ much smaller than the actual gap $h$ the apparent shear rate detected externally by measurements of the boundary motion at a given stress can be written as

$$
\dot{\gamma}=\dot{\gamma}_{b}+\frac{2 \delta}{h} \dot{\gamma}_{w}, \quad \delta \ll h
$$

Linear regression of plots of shear rate as a function of the reciprocal of the gap $h$ for constant values of the shear stresses enable the determination of the gradient, also known as the slip velocity $v_{s}=2 \delta \dot{\gamma}_{w}$. The variation of this slip velocity with stress can typically be represented in a power law form, $v_{s}=\beta \tau^{m}$. The value $m=1$ corresponds to the Navier slip law. Although 
such experiments and analysis enable one to assess if slip is occurring in a sample, they do not allow one to determine if the slip occurs on both walls with each layer of thickness $\delta$ or in a single localized layer or 'shear band' of thickness $2 \delta$ somewhere within the sample.

A slip analysis of the data from Figure 5, based on the formulation of eq. (2), is shown in Figure 6. At each value of the shear stress, $\tau$, two different slip regions can be discerned from the data. The thick solid line in Figure 6 approximately demarcates two regimes which have different slopes. Linear regression of each data set for a constant stress, thus results in a different slip velocity in each region. These two slip velocities, denoted $v_{s}^{I}$ and $v_{s}^{I I}$ are plotted as a function of the applied shear stress in Figure 7. Below a stress of $\tau_{s, \min } \sim 380$ Pa no slip can be detected by our instrument. As the stress is incremented towards a value of $540 \mathrm{~Pa}$ only the first slip region exists and the approximately linear variation of the slip velocity with shear stress on this doublelogarithmic plot suggests that, over this range of stresses at least, it is proportional to the stress raised to a power of 4.4. Above a gap-dependent critical stress level $\tau_{s}^{I I}(h)$, however, the type of slip changes, the slip velocity jumps to a higher absolute value but is now proportional to the stress with a power of 1.8. The stress level at which this transition occurs is nearly constant for gaps $h \geq$ $55 \mu \mathrm{m}$ at $\tau_{s}^{I I} \rightarrow 540 \mathrm{~Pa}$.

Different scaling of the slip velocity with the stress in different regimes has previously been reported for polymer melts and solutions [24, 42], also an abrupt increase of the slip velocity has been noted at the transition between two different slip mechanisms [43]. The new observation in this microrheometer study is that for gaps below $55 \mu \mathrm{m}$ the stress level at which this transition between the two slip regions occurs depends on the gap. Figure 7 shows that the stress level of the transition is shifted to higher values with a decreasing gap. At the same time the magnitude of the abrupt jump in the slip velocity decreases with decreasing gap. An extrapolation of the slip velocity - stress data for the slip region I to high stress values in Figure 7 indicates that at a critical stress of $850 \mathrm{~Pa}$ and for very small gaps close to the zero-gap limit a smooth transition from slip type I to slip type II occurs without a jump in slip velocity. This gap-dependent transition is in accordance with the gradual change of the "pseudo yielding" of the third yield region from a clear transition at large gaps to a mere inflexion point at small gaps (cf. Figure 5).

The critical stress levels documented for each different gap-dependent microrheological phenomena can be compiled into a "phase diagram" for the flow of skin crème at gaps below 
$100 \mu \mathrm{m}$ as shown in Figure 8. The sequence of stress-induced transitions observed in this oil-water emulsion at a given gap separation correspond to vertical trajectories through this phase diagram. At very large gaps, such as those used in a conventional rheometer, there are four regimes: At low stresses the material creeps like an elastoplastic solid, or equivalently a fluid with a very high viscosity [38]. At a critical stress of approximately $\tau_{y} \approx 240 \mathrm{~Pa}$ there is a yielding transition and the material flows viscoplastically. Beyond an almost-gap-independent critical stress of $\tau_{s \text {,min }}$ $\approx 380 \mathrm{~Pa}$ the flowing material starts to slip against the plates and at higher stresses beyond $\tau_{s}^{I I}(h)$ there is a (gap dependent) transition to a second slip mechanism. For 'large' gaps above $100 \mu \mathrm{m}$ it appears that $\tau_{s}^{I I}(h) \rightarrow 540 \mathrm{~Pa}$.

However as the gap is decreased below $100 \mu \mathrm{m}$, it becomes clear that the primary viscoplastic yielding transition occurs at progressively higher stresses. This critical stress, $\tau_{y}(h)$, eventually intersects with the critical stress $\tau_{s, \text { min }}$ for onset of slip. Optical microscopy images of samples of the skin crème studied in these experiments are shown in Figure 9 and can also provide some insight for the observed microscale flow properties. It can be seen from Figure 9a that the skin crème contains solid particles of microcrystalline wax, with the larger particles having mean sizes on the order of $\sim 50 \mu \mathrm{m}$. This particle size correlates very well with the critical gap of $55 \mu \mathrm{m}$, above which the maximum primary yield stress is observed. The increasing yield stress with a decreasing gap is therefore not caused by the emulsion phases of the sample, since the average droplet size of the emulsion is much smaller than the critical gap of $55 \mu \mathrm{m}$ [23], but by the increasing interactions of the two walls via discrete wax particles located near opposing walls.

For gaps below $55 \mu \mathrm{m}$ and stresses below $400 \mathrm{~Pa}$ the flow behavior is dominated by the plastic deformation of individual wax particles squeezed between the shearing surfaces. Plastic deformation of the stressed wax particles, possibly combined with a discontinuous stick slip rolling mechanism between the plates, leads to the increased temporal fluctuations of the stress signal in this area of the phase diagram and disturbs the smooth viscoplastic creep of the sample that is observed at low stresses for gaps $h>55 \mu \mathrm{m}$. The mean wax particle size of approximately $50 \mu \mathrm{m}$ also correlates with the gap setting characterizing the onset of the rise in the critical stress value separating the borderline between the two slip regions. Although the nature of the two slip regions cannot be quantified since the composition of the skin crème is not completely known, it can be assumed that the trapped and compressed wax particles which are present below a gap of $50 \mu \mathrm{m}$ 
result in an increasing normal force on the plates and an increased frictional resistance to slip. This

therefore raises the stress value $\tau_{s}^{I I}(h)$ for the onset of the second slip region. Finally, as shown in the 'phase diagram' in Figure 8 by the hollow symbols, the values for the third pseudo yield region obtained from Figure 5 nearly match the bounding line of the two slip regions in Figure 8, suggesting that this transition boundary really refers to a structural change of the flow in the slip boundary layer.

\section{Conclusions}

In this paper we have documented the complex microrheology that may arise in a typical consumer product such as a skin crème, when the microstructure of the multiphase material (in this case a oil-water emulsion containing microcrystalline wax particles) and the characteristic length scale of the flow device become comparable. A sequence of flow transitions may occur leading to an apparent viscosity that is both gap- and shear-stress- (or shear-rate)-dependent. These transitions can be represented most clearly in the form of a 'phase diagram' identifying the critical stress for each transition as a function of the gap. Combining microrheometry and optical microscopy can further aid in identifying the probable microscopic mechanisms for each change in the fluid response. In the case of the skin crème investigated in the present study, although it consists primarily of an emulsion, the flow behavior in small gaps below $100 \mu \mathrm{m}$ is dominated by the micro-crystalline wax particle component.

By combining the micro-rheological measurements with a slip analysis of the type described first by Mooney and subsequently by Yoshimura \& Prud'homme, additional insight can also be obtained. The sudden increase in the slip velocity between two surfaces with decreasing gap and increasing applied stress that is shown in Figure 7 is of importance in end-user perceptions of textural quantities such as 'slipperiness' or 'lubricity' or 'skin feel' which are typically used to characterize creams and other complex consumer products [26].

A number of different microrheological techniques are currently under development in many laboratories for different applications. We believe that our ability to steadily vary the gap between the two shearing surfaces means that the flexure-based microrheometer developed in the present work is likely to find considerable application in the future in helping bridge the gap 
between the two traditionally-distinct fields of bulk rheological characterization and tribological testing.

\section{Acknowledgments}

The authors would like to thank Prof. L. Anand, and B. Gearing in the Dept. of Mechanical Engineering at MIT for their kind support with the design of a compound spring and the DupontMIT-Alliance (DMA) for financial support.

\section{References}

1. C.F. Brooks, G.G. Fuller, C.W. Frank, and C.R. Robertson, An interfacial stress rheometer to study rheological transitions in monolayers at the air-water interface, Langmuir, 15 (1999) 2450.

2. T. Richards, Interfacial rheology: Principle, applications, and instrumentation, American Laboratory, 33 (2001) 38.

3. D.A. Edwards, H. Brenner, and D.T. Wasan, Interfacial Transport Processes and Rheology, Butterworth-Heinemann: Boston. 1991.

4. B. Bhushan, Fundamentals of Tribology and Bridging the Gap Between the Macro and Micro/Nanoscales, in: NATO/ASI Science Series: Mathematics, Physics and Chemistry, vol 10, Kluwer: Boston. 2000.

5. M.A. Green and C.A. Brockley, Viscoelastic Effects in Boundary Lubrication, Nature, 251 (1974) 306.

6. Y.-Z. Hu and S. Granick, Microscopic Study of Thin Film Lubrication and its Contributions to Macroscopic Tribology, Tribology Letters, 5 (1998) 81.

7. A. Mukhopadhyay and S. Granick, Micro- and nanorheology, Current Opinion in Colloid \& Interface Science, 6 (2001) 423.

8. F.C. MacKintosh and C.F. Schmidt, Microrheology, Current Opinion in Colloid \& Interface Science, 4 (1999) 300.

9. M.J. Solomon and Q. Lu, Rheology and dynamics of particles in viscoelastic media, Current Opinion in Colloid \& Interface Science, 6 (2001) 430.

10. D.A. Weitz and D.J. Pine, Diffusing Wave Spectroscopy, in: W. Brown (Eds.), Dynamic Light Scattering, OUP: Oxford. 1992.

11. G. Popescu, A. Dogariu, and R. Rajagopalan, Spatially resolved microrheology using localized coherence volumes, Physical Review E, 65 (2002) art. no. 041504.

12. J.L. McGrath, J.H. Hartwig, and S.C. Kuo, The mechanics of F-actin microenvironments depend on the chemistry of probing surfaces (vol 79, pg 3258, 2000), Biophysical Journal, 80 (2001) 2487.

13. A.J. Levine and T.C. Lubensky, One- and two-particle microrheology, Physical Review Letters, 85 (2000) 1774. 
14. A.J. Levine and T.C. Lubensky, Response function of a sphere in a viscoelastic two-fluid medium, Physical Review E, 6304 (2001) art. no. 041510.

15. T.G. Mason, Estimating the viscoelastic moduli of complex fluids using the generalized Stokes-Einstein equation, Rheologica Acta, 39 (2000) 371.

16. J. Choi and T. Kato, Static and dynamic behavior of liquid nano-meniscus bridge (c), Tribology Transactions, 44 (2001) 19.

17. P.R. Williams, Videos of microcavitation using an AFM tip, (2002).

18. C. Ortiz and Hadziioannou, Entropic Elasticity of Single Polymer Chains of Poly(methacrylic acid) Measured by Atomic Force Microscopy, Macromolecules, 32 (1999) 780.

19. A.V. Bazilevskii, V.M. Entov, and A.N. Rozhkov, Breakup of an Oldroyd liquid bridge as a method for testing the rheological properties of polymer solutions, Polymer Science Series A, 43 (2001) 716.

20. S.L. Anna and G.H. McKinley, Elasto-capillary thinning and breakup of model elastic liquids, Journal of Rheology, 45 (2001) 115.

21. N. Kojic, C. Clasen, J. Bico, and G.H. McKinely, Ex-Vivo Rheology of Spider Silk, Nature Materials, (2003) submitted.

22. S. Boskovic, J.W.M. Chon, P. Mulvaney, and J.E. Sader, Rheological measurements using microcantilevers, Journal of Rheology, 46 (2002) 891.

23. C. Clasen and G.H. McKinley, Microrheology - The Flexure-based Microgap Rheometer (FMR), Journal of Rheology, (2003) submitted.

24. S.G. Hatzikiriakos and J.M. Dealy, Wall Slip of Molten High-Density Polyethylene .1. Sliding Plate Rheometer Studies, Journal of Rheology, 35 (1991) 497.

25. A. Dhinojwala and S. Granick, Micron-gap rheo-optics with parallel plates, Journal of Chemical Physics, 107 (1997) 8664.

26. R. Brummer and S. Godersky, Rheological studies to objectify sensations occurring when cosmetic emulsions are applied to the skin, Colloids and Surfaces a-Physicochemical and Engineering Aspects, 152 (1999) 89.

27. M.M.J. Soriano, M.J.F. Contreras, and E.S. Flores, Development of a cream from a selfemulsifying base and moisturizing actives, Farmaco, 56 (2001) 513.

28. G.H. Meeten, Yield stress of structured fluids measured by squeeze flow, Rheologica Acta, 39 (2000) 399.

29. G.H. Meeten, Squeeze flow between plane and spherical surfaces, Rheologica Acta, 40 (2001) 279.

30. G.H. Meeten, Constant-force squeeze flow of soft solids, Rheologica Acta, 41 (2002) 557.

31. B.P. Gearing and L. Anand. in ASME Int. Mech. Eng. Congress and Exposition. 2001. New York, NY.

32. G.J.C. Braithwaite and G.H. McKinley, Microrheometry for Studying the Rheology and Dynamics of Polymers near Interfaces, Applied Rheology, 9 (1999) 27.

33. P.V. Pavoor, B.P. Gearing, A. Bellare, and R.E. Cohen, Tribology Characteristics of Polyectrolyet Multilayers, Wear, (2003) submitted. 
34. G. Luengo, J. Israelachvili, and S. Granick, Generalized Effects in Confined Fluids: new Friction Map for Boundary Lubrication, Wear, 200 (1996) 328.

35. G.M. Eccleston, Functions of mixed emulsifiers and emulsifying waxes in dermatological lotions and creams, Colloids and Surfaces a-Physicochemical and Engineering Aspects, 123 (1997) 169.

36. B.W. Barry and G.M. Saunders, The Self-Bodying Action of the Mixed Emulsifier Cetrimide Cetostearyl Alcohol, Journal of Colloid and Interface Science, 34 (1970) 300.

37. M. Korhonen, H. Niskanen, J. Kiesvaara, and J. Yliruusi, Determination of optimal combination of surfactants in creams using rheology measurements, International Journal of Pharmaceutics, 197 (2000) 143.

38. H.A. Barnes, The yield stress - a review or 'pi alpha nu tau alpha rho epsilon iota' everything flows?, Journal of Non-Newtonian Fluid Mechanics, 81 (1999) 133.

39. H.A. Barnes, A Review of the Slip (Wall Depletion) of Polymer-Solutions, Emulsions and Particle Suspensions in Viscometers - Its Cause, Character, and Cure, Journal of Non-Newtonian Fluid Mechanics, 56 (1995) 221.

40. A. Yoshimura and R.K. Prudhomme, Wall Slip Corrections for Couette and Parallel Disk Viscometers, Journal of Rheology, 32 (1988) 53.

41. Y. Cohen and A.B. Metzner, Apparent Slip-Flow of Polymer-Solutions, Journal of Rheology, 29 (1985) 67.

42. L. Devargas and O. Manero, On the Slip Phenomenon of Polymeric Solutions through Capillaries, Polymer Engineering and Science, 29 (1989) 1232.

43. V. Mhetar and L.A. Archer, Slip in entangled polymer solutions, Macromolecules, 31 (1998) 6639. 


\section{FIGURES}

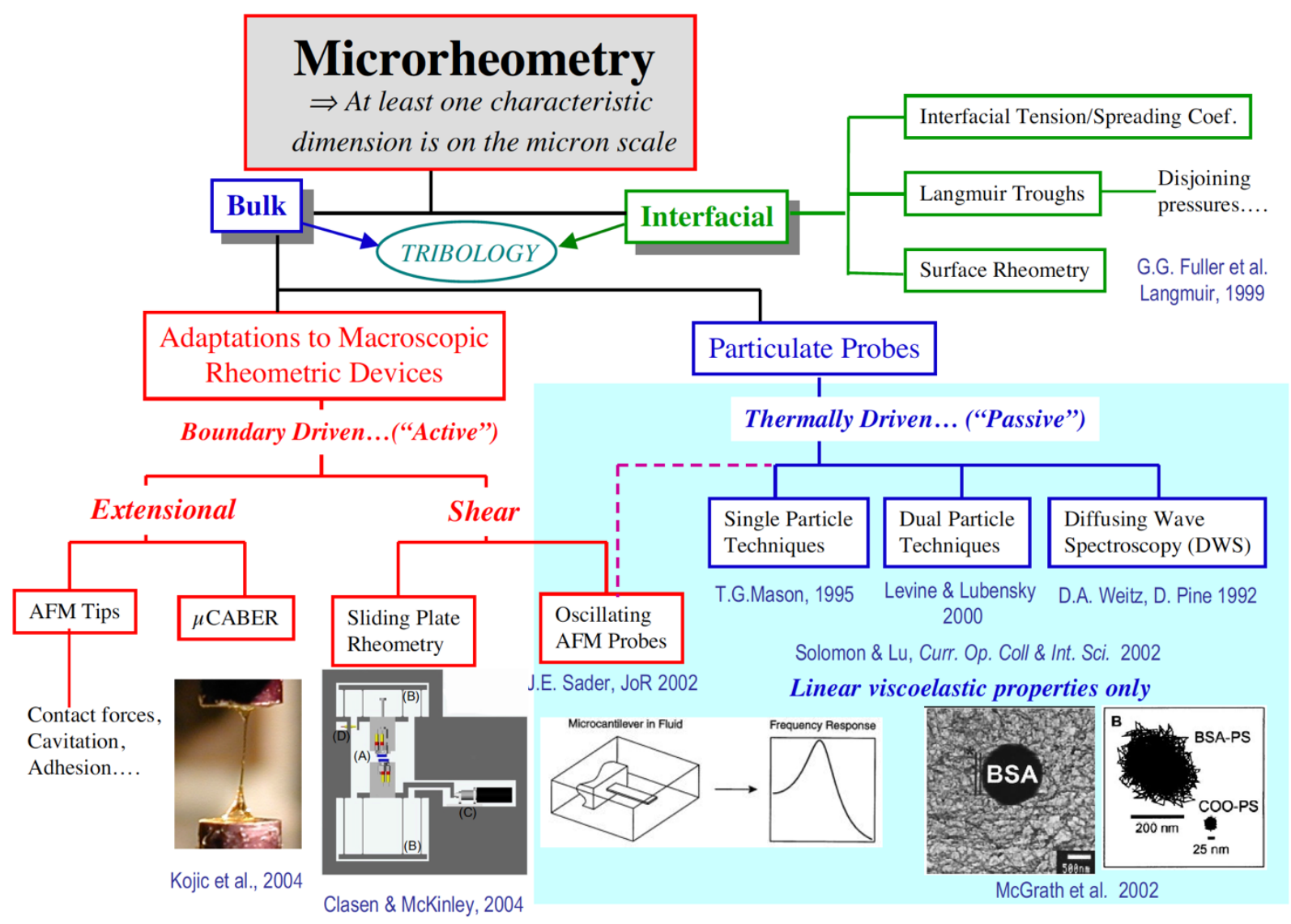

Figure 1

A summary of recent developments in the field of Microrheology 


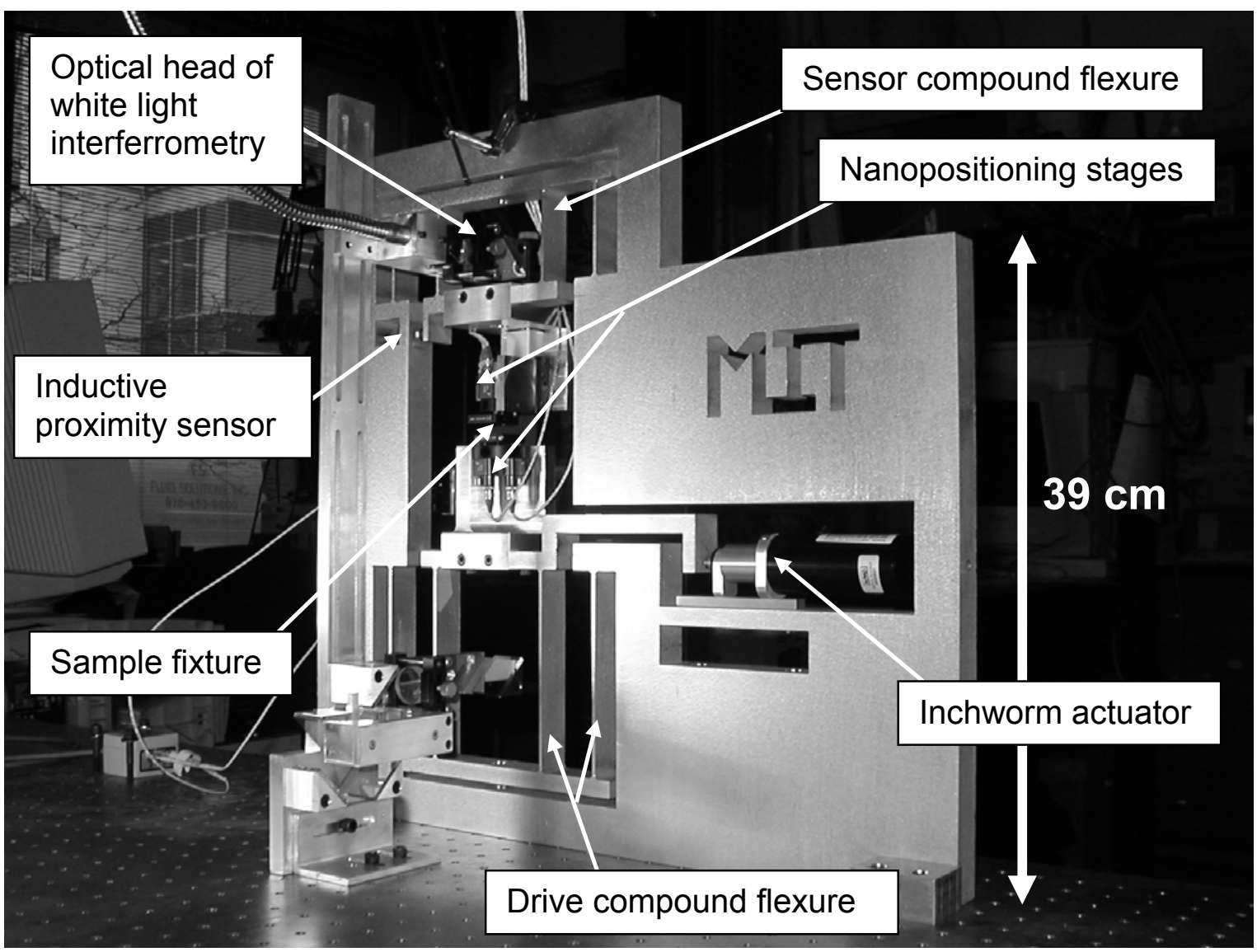

Figure 2

The Flexure-based Microgap Rheometer (FMR) showing a picture of the assembled instrument with the white light interferometry unit in the foreground. The instrument frame is approximately $30 \mathrm{~cm}$ tall. 


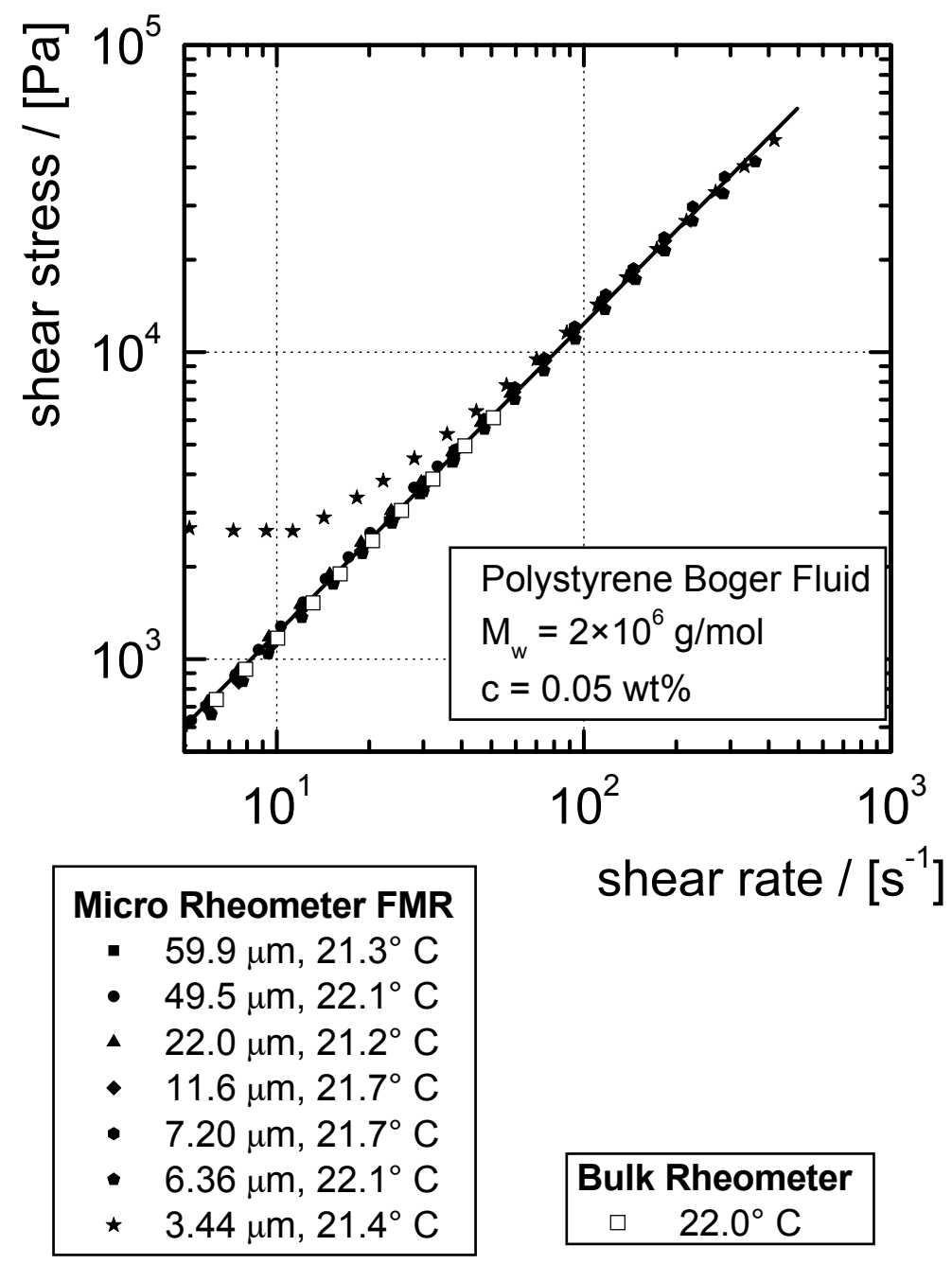

Figure 3

Comparison of the shear stress as function of the shear rate for different gaps on the Flexure based Microgap Rheometer (FMR) (closed symbols) and a bulk rheometer (open symbols) for a Boger fluid consisting of $250 \mathrm{ppm}$ of high molecular polystyrene $\left(2.25 \times 10^{6} \mathrm{~g} / \mathrm{mol}\right)$ dissolved in oligomeric styrene. 


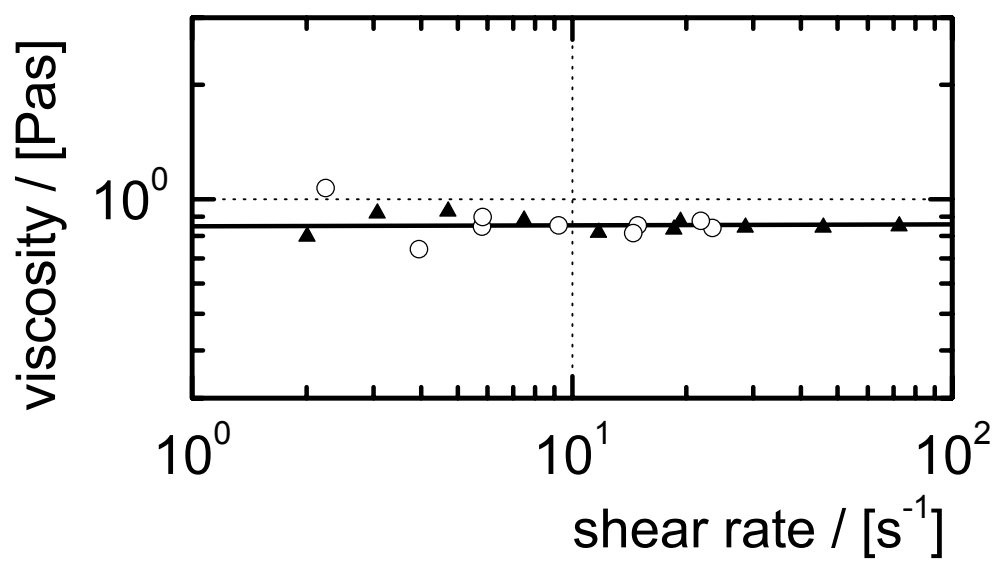

Figure 4

Viscosity as a function of the shear rate for an unentangled polydimethyl siloxane (PDMS) standard with a nominal viscosity of $1000 \mathrm{cSt}$. The data is measured using the Flexure Based Microgap Rheometer (FMR) with two different gaps (open circles (o): $88.8 \mu \mathrm{m}, 23.8{ }^{\circ} \mathrm{C}$, closed triangles $(\boldsymbol{\Delta}): 25.4 \mu \mathrm{m}, 23.8^{\circ} \mathrm{C}$ ) 


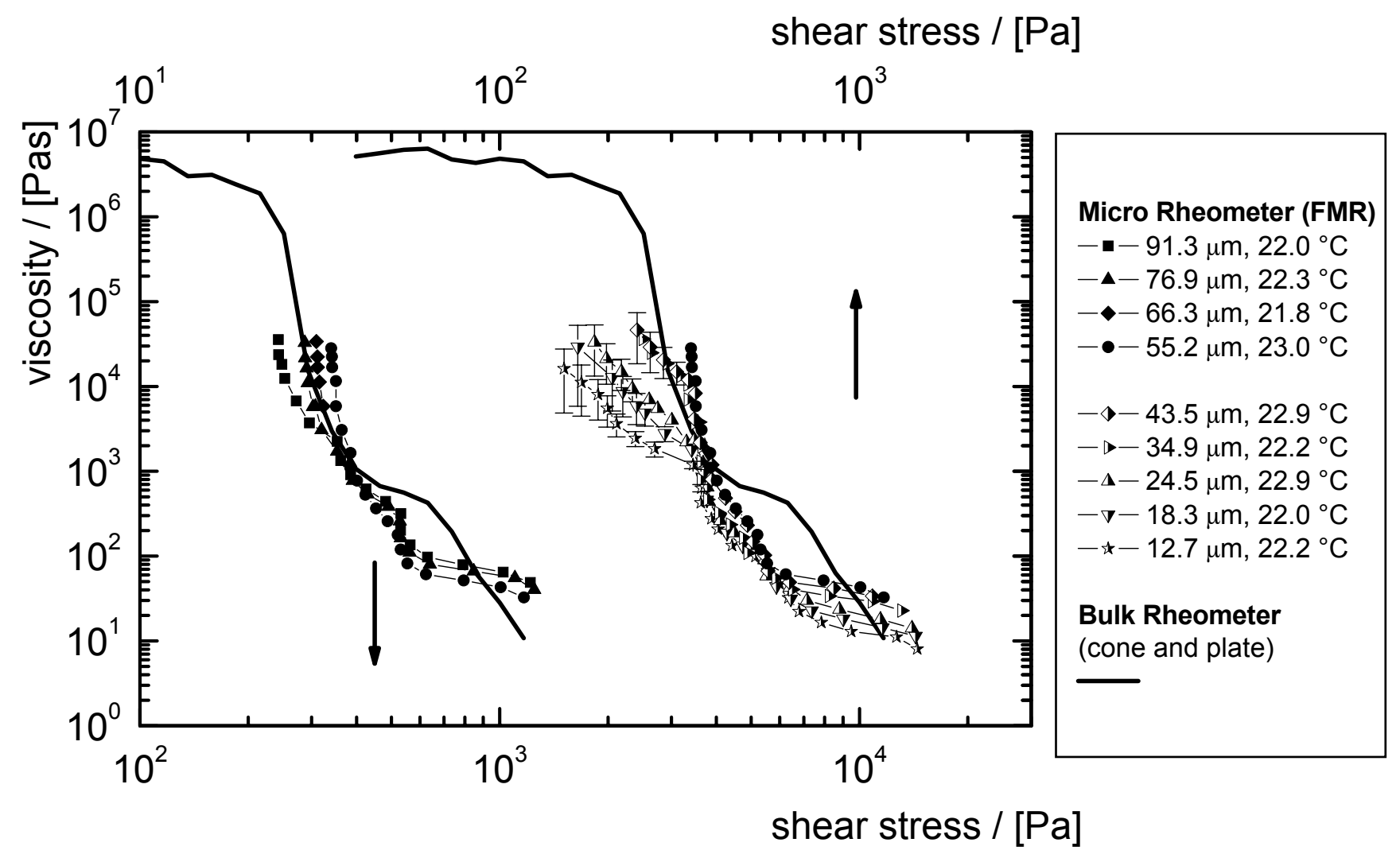

Figure 5

Apparent viscosity as a function of the shear stress for skin crème. The solid lines refer to the bulk rheological data, the symbols indicate the data obtained from the FMR. The conical fixture has a cone angle of $\theta_{0}=1^{\circ} 59^{\prime}$ and a truncation of $56 \mu \mathrm{m}$. The results are split between both an upper and a lower abscissa as indicated for better visualization. The data for the bulk rheology and for a gap of $h=55.2 \mu \mathrm{m}$ are shown on both stress axes for reference. 


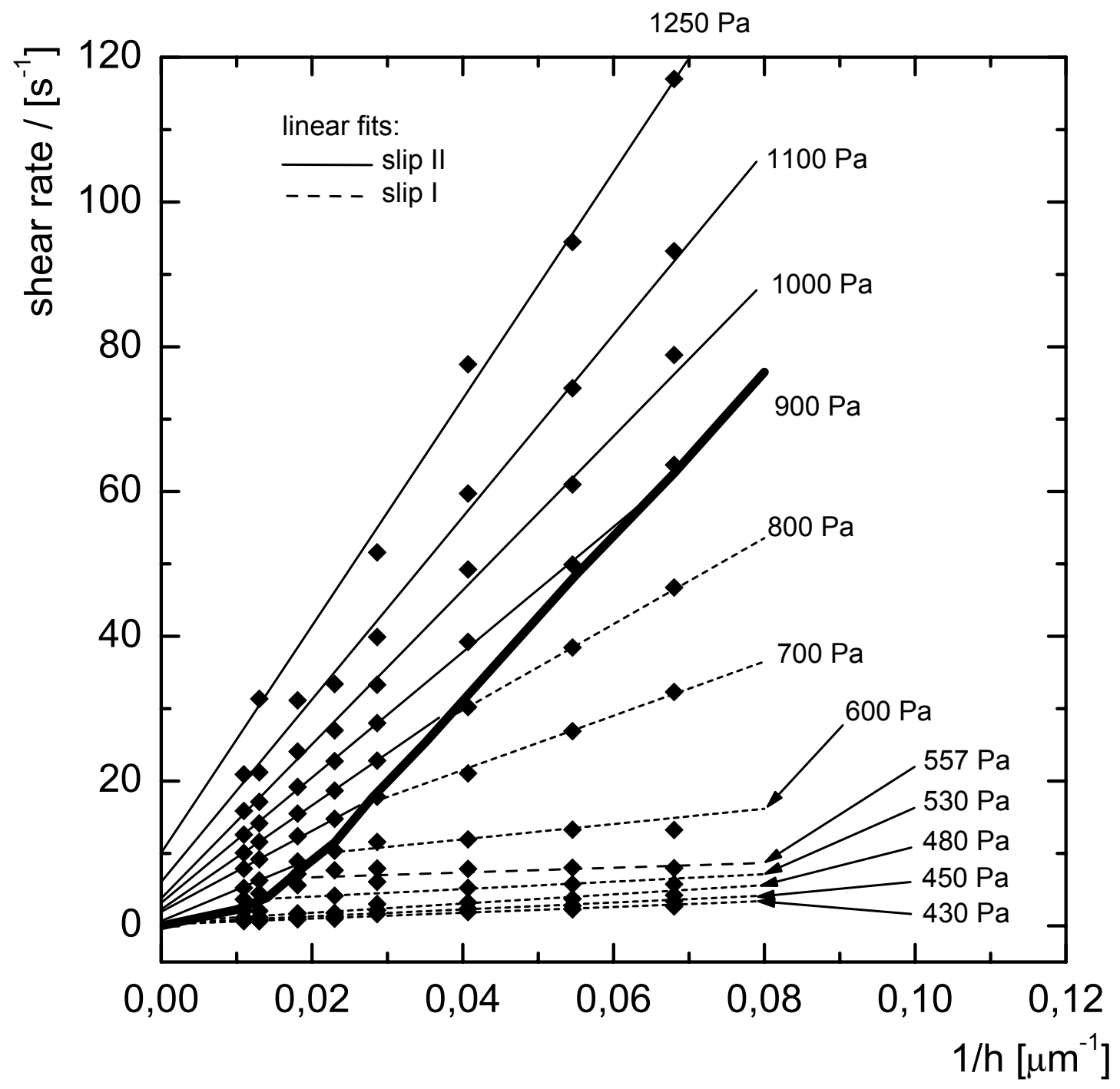

Figure 6

Apparent shear rate $\dot{\gamma}$ as a function of the reciprocal gap 1/h according to Eq. (2), data taken from figure 5. The straight line separates two regimes with different slopes of the linear fits for a constant stress. 


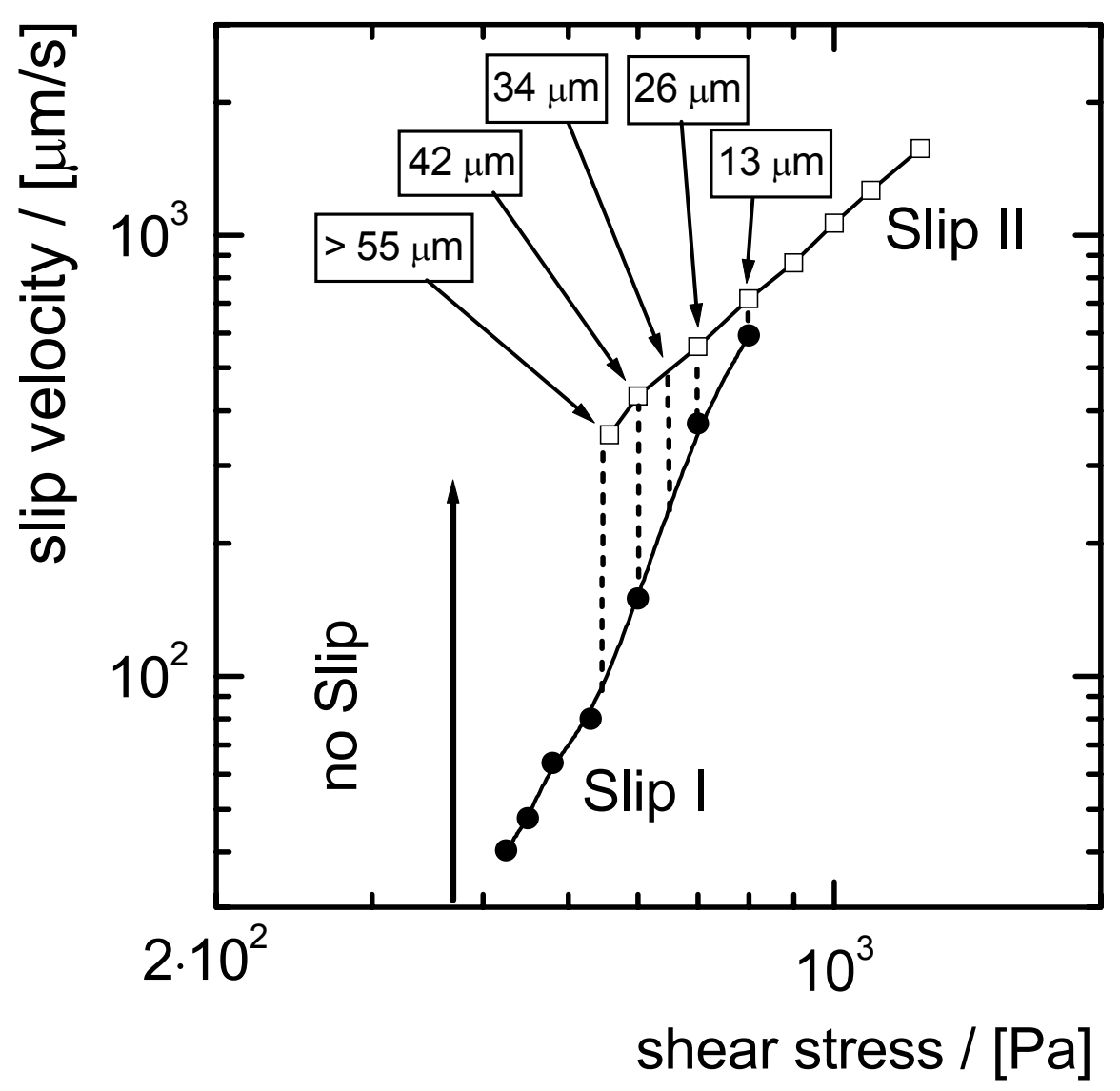

Figure 7

Slip velocities as a function of the stress. The data can be represented as power laws of the form $v_{s}^{i}=\beta^{i} \tau^{m_{i}}$ with $i=I$, II. Regression gives for slip mechanism $\mathrm{I} ; \beta^{I}=1.01 \times 10^{-10}(\mu \mathrm{m} / \mathrm{s})\left(\mathrm{Pa}^{-\mathrm{m}}\right)$, $m_{I}=4.40$; for slip mechanism II; $\beta^{I I}=3.91 \times 10^{-3}(\mu \mathrm{m} / \mathrm{s})\left(\mathrm{Pa}^{-\mathrm{m}}\right), m^{I I}=1.81$. The critical value of the stress for transition between the slip velocities of the two types of slip observed in Figure 6 depends on the gap for gaps below $55 \mu \mathrm{m}$. 


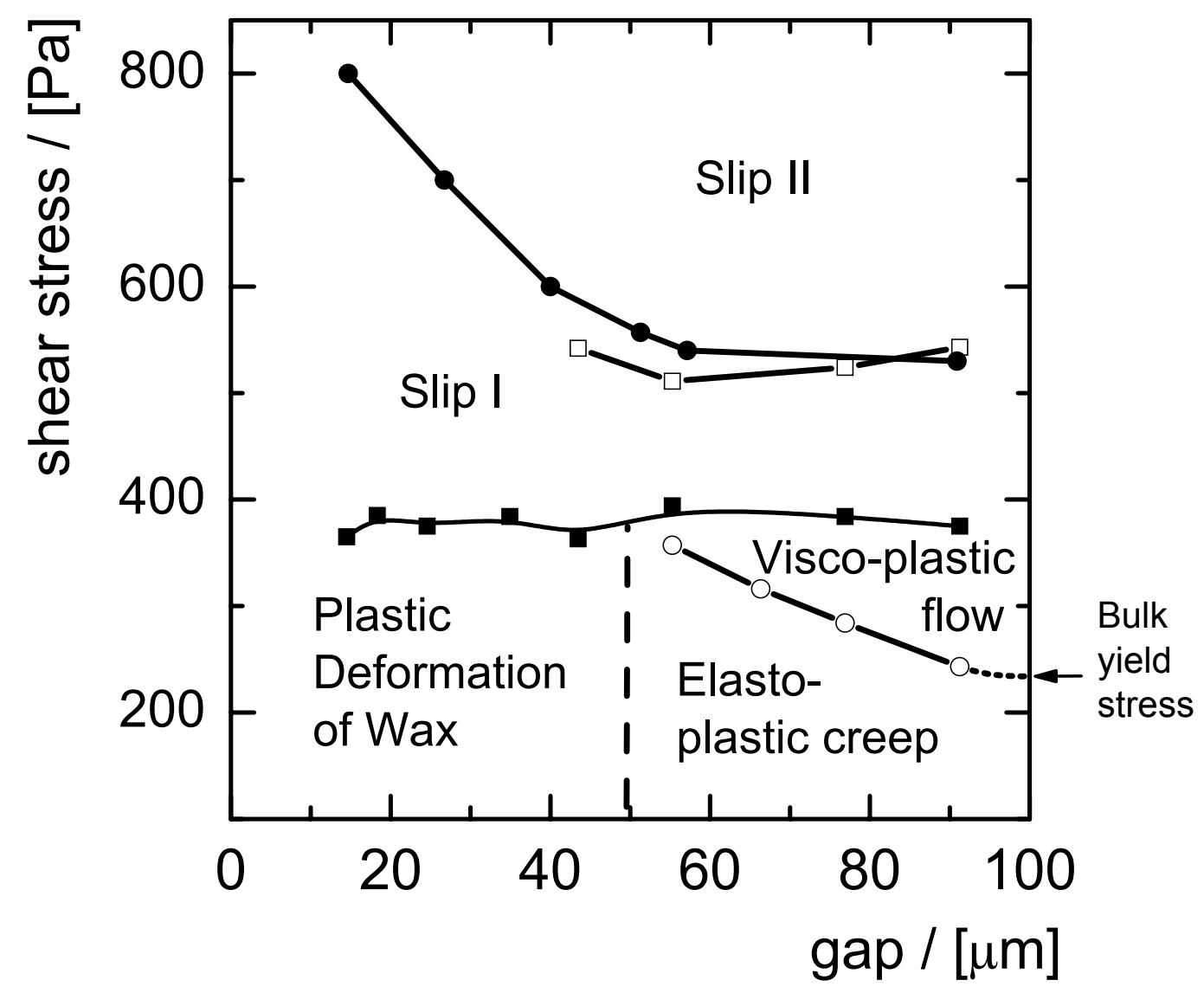

Figure 8

"Phase diagram" of the gap dependent microrheology of skin crème as a function of the applied shear stress and the gap separation. The hollow circles $(\circ)$ refer to the primary yielding stress for gaps above $50 \mu \mathrm{m}$, the filled squares ( $\mathbf{m})$ refer to the gap independent secondary yield stress and the open squares $(\square)$ refer to the stress values of the third yielding region for gaps above $50 \mu \mathrm{m}$, all values taken from Figure 5. The filled circles $(\bullet)$ refer to the stress values of the solid line in Figure 6. 

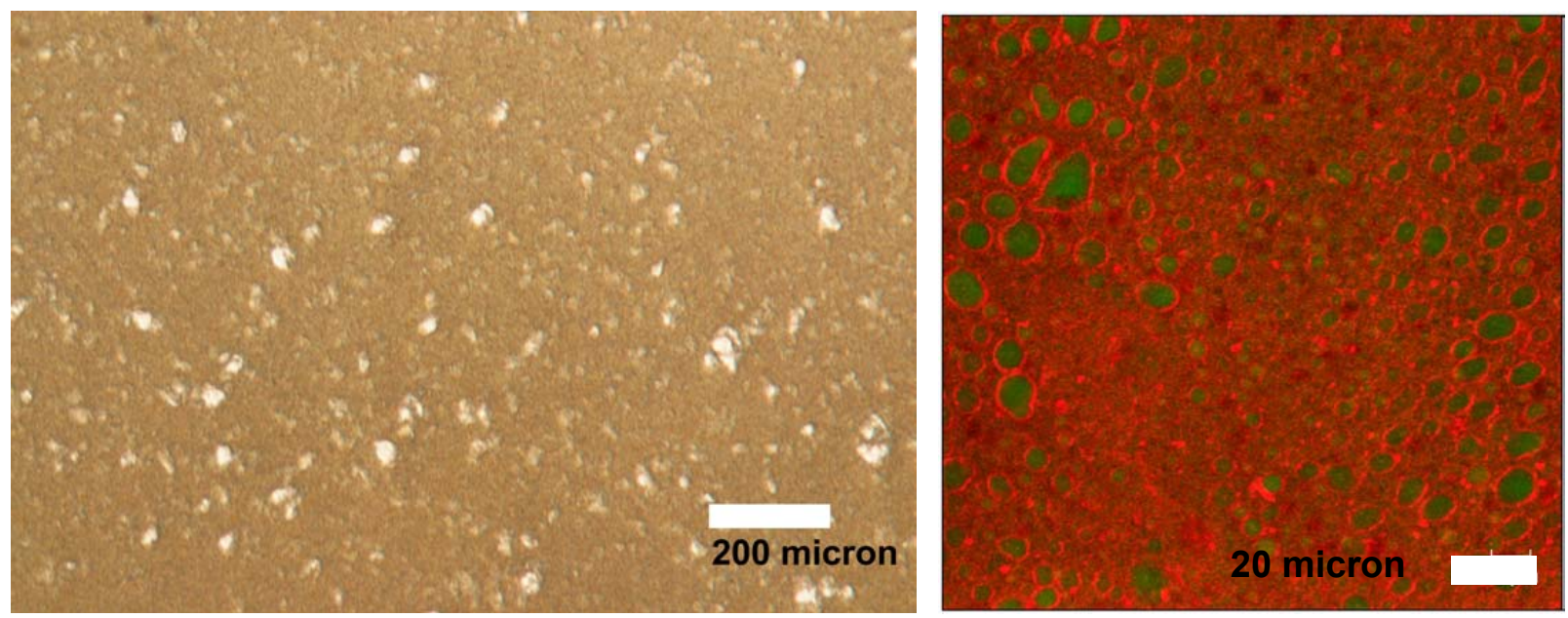

Figure 9

Microscopic view of the skin cream at two different magnifications, the viewing direction is perpendicular to the gap. 